\title{
WORD LENGTH AND LIMIT SETS OF KLEINIAN GROUPS
}

\author{
TSUYOSHI YAMAGUCHI \\ Dedicated to the memory of Professor Nobuyuki Suita
}

\section{Introduction}

For the real or complex hyperbolic space $\mathbf{H}^{n}$, a discrete subgroup of the isometry group Isom ${ }^{+} \mathbf{H}^{n}$ is called a Kleinian group. It is well known that a Kleinian group $G$ acts properly discontinuously on the hyperbolic space $\mathbf{H}^{n}$. Hence, the orbit $G(o)$ of a point $o \in \mathbf{H}^{n}$ has accumulation points, which are called limit points of $G$, only on the boundary at infinity $\partial \mathbf{H}^{n}$. The set of all limit points is called the limit set of $G$, which is denoted by $\Lambda(G)$. The limit set $\Lambda(G)$ plays an important role in studying the Kleinian group $G$ and the hyperbolic 3-manifold $\mathbf{H}^{3} / G$.

We may classify limit points of $G$ according to the way how the orbit $G(o)$ accumulates to the limit point. For example, if $z \in \partial \mathbf{H}^{n}$ is a fixed point of a loxodromic transformation $g \in G$, then the orbit $\left\{g^{n}(o)\right\}$ converges to $z$ inside a cone around the axis of $g$. On the other hand, if $z^{\prime} \in \partial \mathbf{H}^{n}$ is the fixed point of a parabolic transformation $h \in G$, then the orbit $\left\{h^{n}(o)\right\}$ converges to $z^{\prime}$ on a horosphere in $\mathbf{H}^{n}$. Comparing those two cases, we find that the growth rate of the hyperbolic distances $d_{h}\left(o, g^{n}(o)\right)$ is much larger than that of $d_{h}\left(o, h^{n}(o)\right)$ as $n \rightarrow \infty$. Therefore, it is expected that the growth rate of the orbit would be crucial in the theory of Kleinian groups. From this point of view, we introduce the following quantity $\alpha(G)$ for a finitely generated Kleinian group $G$.

Take a generating set $\sigma$ of $G$. Let $|g|$ denote the minimal word length of $g \in G$ with respect to $\sigma$. We define $\alpha(G)$ by

$$
\alpha(G)=\sup \left\{\alpha \mid \sup _{g \in G}\left\{\frac{|g|^{\alpha}}{e^{d_{h}(o, g(o))}}\right\}<+\infty\right\} .
$$

In [5], W. J. Floyd considered a relationship between Cayley graphs and limit sets of Kleinian groups. In the discussion, he showed that for a geometrically finite group $G, \alpha(G)=+\infty$ if $G$ has no parabolic transformations while $\alpha(G)=2$ if it contains a parabolic transformation. It is also shown that $\alpha(G) \leq 2$ for any finitely generated Kleinian group $G$ with parabolic transformations. 
In this paper, we shall show that $\alpha(G)>2$ always implies $\alpha(G)=+\infty$ and the geometric finiteness of $G$. Namely, the following is our main theorem.

THEOREM 4.2. Let $G$ be a finitely generated Kleinian group. Then, the following conditions are equivalent:

1. $G$ is convex co-compact;

2. $\alpha(G)>2$;

3. $\alpha(G)=+\infty$.

From this theorem, immediately we have;

COROLlary 4.2. Let $G$ be convex co-compact and $H$ a geometrically infinite group. Suppose that there exists an isomorphism $\phi: G \rightarrow H$. Then, for any $\varepsilon>0$, there exists a $g \in G$ such that $d_{h}(o, \phi(g)(o))<(2+\varepsilon) \log d_{h}(o, g(o))$.

Remark. The existence of the isomorphism as above is guaranteed in many cases. In fact, it is known (cf. [8]) that if $H$ is a finitely generated Kleinian group acting on $\mathbf{H}_{\mathbf{R}}^{3}$ and if the quotient $\mathbf{H}_{\mathbf{R}}^{3} / H$ has infinite volume, then there exist a geometrically finite Kleinian group $G$ and a type-preserving isomorphism $\phi$ of $H$ onto $G$.

In Section 2, we explain the fundamental facts on the real and complex hyperbolic spaces as well as on the limit sets of Kleinian groups and geometrically finite groups. In Section 3, we discuss a map from the group completion $\partial G$ to the limit set $\Lambda(G)$, due to Floyd [5]. In Section 4, we study a relation of the word length and the limit sets, and prove the main theorem.

The author would like to thank Professors H. Shiga and A. Ushijima for numerous advices and encouragement, and thank the referee of an earlier version for suggestions of the improved version of Lemma 4.1.

\section{Hyperbolic geometry}

We will explain fundamental facts on the hyperbolic geometry. For details, see Apanasov [1], Apanasov-Xie [3], Epstein [4], Goldman [6], MatsuzakiTaniguchi [8].

\subsection{Real hyperbolic space}

We denote by $\mathbf{B}_{\mathbf{R}}^{n}$ the unit ball in $\mathbf{R}^{n}$ and by $\partial \mathbf{B}_{\mathbf{R}}^{n}$ the unit sphere in $\mathbf{R}^{n}$. The hyperbolic metric is denoted by $d s$ and is derived from the differential form

$$
d s^{2}=\frac{4|d x|^{2}}{\left(1-|x|^{2}\right)^{2}} .
$$

The resulting metric space $\left(\mathbf{B}_{\mathbf{R}}^{n}, d s\right)$ is called the $n$-dimensional real hyperbolic space $\mathbf{H}_{\mathbf{R}}^{n}$ (of ball model). In this case, $\partial \mathbf{B}_{\mathbf{R}}^{n}$ is denoted by $\partial \mathbf{H}_{\mathbf{R}}^{n}$. The geodesics 
are arcs of Euclidean circles orthogonal to $\partial \mathbf{H}_{\mathbf{R}}^{n}$. The horosphere through a point $u \in \mathbf{H}_{\mathbf{R}}^{n}$ centered at a point $y \in \partial \mathbf{H}_{\mathbf{R}}^{n}$ is the Euclidean sphere which is tangent to $\partial \mathbf{H}_{\mathbf{R}}^{n}$ at $y$ and passes through $u$. We denote by $\operatorname{Isom}^{+} \mathbf{H}_{\mathbf{R}}^{n}$ the group of all orientation-preserving automorphisms of $\mathbf{H}_{\mathbf{R}}^{n}$ that are isometric with respect to the hyperbolic metric.

We define $J: \overline{\mathbf{R}}^{n} \rightarrow \overline{\mathbf{R}}^{n}$ by $J(p)=p /|p|^{2}, J(0)=\infty$ and $J(\infty)=0 . \quad$ Let $e=$ $(0, \ldots, 0,1) \in \mathbf{R}^{n}$ and define a mapping on $\mathbf{R}^{n} \Pi$ by $\Pi(p)=e+2 J(J(p)+e)$. Then we have $\Pi\left(\mathbf{B}_{\mathbf{R}}^{n}\right)=\mathbf{R}_{+}^{n}$, where $\mathbf{R}_{+}^{n}=\left\{x=\left(x_{1}, \ldots, x_{n}\right) \in \mathbf{R}^{n} \mid x_{n}>0\right\}$. By this transformation, $\mathbf{H}_{\mathbf{R}}^{n}$ is identified with $\mathbf{R}_{+}^{n}$, and the metric in $\mathbf{R}_{+}^{n}$ is

$$
d s^{2}=\frac{|d x|^{2}}{x_{n}^{2}} .
$$

The upper half-space $\mathbf{R}_{+}^{n}$ is called the upper half-space model of the real hyperbolic space. $\partial \mathbf{H}_{\mathbf{R}}^{n}$ is identified with $\{\infty\} \cup\left\{x \in \mathbf{R}^{n} \mid x_{n}=0\right\}$. The horosphere through a point $u \in \mathbf{R}_{+}^{n}$ centered at a point $y \in\left\{x \in \mathbf{R}^{n} \mid x_{n}=0\right\}$ is the Euclidean sphere which is tangent to $\left\{x \in \mathbf{R}^{n} \mid x_{n}=0\right\}$ at $y$ and passes through $u$, and, the horosphere through $u=\left(u_{1}, \ldots, u_{n-1}, t\right) \in \mathbf{R}_{+}^{n}(t>0)$ centered at $\infty$ is the set $\left\{x \in \mathbf{R}^{n} \mid x_{n}=t\right\}$.

\subsection{Complex hyperbolic space}

We denote by $\mathbf{B}_{\mathbf{C}}^{n}$ the unit ball in $\mathbf{C}^{n}$ and by $\partial \mathbf{B}_{\mathbf{C}}^{n}$ the unit sphere in $\mathbf{C}^{n}$. The hyperbolic metric is denoted by $d s$ and is derived from the differential form

$$
|d s|^{2}=\frac{4}{(1-\langle\langle z, z\rangle\rangle)^{2}}\{\langle\langle z, d z\rangle\rangle\langle\langle d z, z\rangle\rangle+(1-\langle\langle z, z\rangle\rangle)\langle\langle d z, d z\rangle\rangle\},
$$

where $\langle\langle z, z\rangle\rangle=z_{1} \bar{z}_{1}+\cdots+z_{n} \bar{z}_{n}$. The resulting metric space $\left(\mathbf{B}_{\mathbf{C}}^{n}, d s\right)$ is called the $n$-dimensional complex hyperbolic space (of projective model), which denoted by $\mathbf{H}_{\mathbf{C}}^{n}$. In this case, $\partial \mathbf{B}_{\mathbf{C}}^{n}$ is regarded as $\partial \mathbf{H}_{\mathbf{C}}^{n}$. The horosphere through $u \in \mathbf{H}_{\mathbf{C}}^{n}$ centered at $y \in \partial \mathbf{H}_{\mathbf{C}}^{n}$ is the set $\{z||(1-\langle\langle z, z\rangle\rangle)(1-\langle\langle u, y\rangle\rangle)|=|(1-$ $\langle\langle u, u\rangle\rangle)(1-\langle\langle z, y\rangle) \mid\}$. We denote by $\operatorname{Isom}^{+} \mathbf{H}_{\mathbf{C}}^{n}$ the group of all orientationpreserving automorphisms of $\mathbf{H}_{\mathbf{C}}^{n}$ that are isometric with respect to the hyperbolic metric.

We define $P: \mathbf{B}_{\mathbf{C}}^{n} \rightarrow \mathbf{C}^{n} \quad$ by $\quad\left(z_{1}, \ldots, z_{n}\right) \mapsto\left(z_{1} /\left(1+z_{n}\right), \ldots, z_{n-1} /\left(1+z_{n}\right)\right.$, $\left.1-z_{n} / 2\left(1+z_{n}\right)\right)$. Then we have $P\left(\mathbf{B}_{\mathbf{C}}^{n}\right)=S^{n}=\left\{z=\left(z^{\prime}, z_{n}\right) \in \mathbf{C}^{n} \mid z_{n}+\bar{z}_{n}>\right.$ $\left.\left\langle\left\langle z^{\prime}, z^{\prime}\right\rangle\right\rangle\right\}$, where $\left\langle\left\langle z^{\prime}, z^{\prime}\right\rangle\right\rangle=z_{1} \bar{z}_{1}+\cdots+z_{n-1} \bar{z}_{n-1}$. By this transformation, $\mathbf{H}_{\mathbf{C}}^{n}$ is identified with $S^{n}$, and the metric in $S^{n}$ is

$$
|d s|^{2}=\frac{4\left(d z_{n}-\left\langle\left\langle d z^{\prime}, z^{\prime}\right\rangle\right\rangle\right)\left(d \bar{z}_{n}-\left\langle\left\langle z^{\prime}, d z^{\prime}\right\rangle\right\rangle\right)+\left(z_{n}+\bar{z}_{n}-\left\langle\left\langle z^{\prime}, z^{\prime}\right\rangle\right\rangle\right)\left\langle\left\langle d z^{\prime}, d z^{\prime}\right\rangle\right\rangle}{\left(z_{n}+\bar{z}_{n}-\left\langle\left\langle z^{\prime}, z^{\prime}\right\rangle\right\rangle\right)^{2}} .
$$

The Siegel domain $S^{n}$ is called the paraboloid model of the complex hyperbolic space. $\quad \partial \mathbf{H}_{\mathbf{C}}^{n}$ is identified with $\{\infty\} \cup\left\{z=\left(z^{\prime}, z_{n}\right) \in \mathbf{C}^{n} \mid z_{n}+\bar{z}_{n}=\left\langle\left\langle z^{\prime}, z^{\prime}\right\rangle\right\rangle\right\}$. The horosphere centered at $\infty$ is the set $\left\{z=\left(z^{\prime}, z_{n}\right) \in \mathbf{C}^{n} \mid z_{n}+\bar{z}_{n}-\left\langle\left\langle z^{\prime}, z^{\prime}\right\rangle\right\rangle=t\right\}$ $(t>0)$. 


\subsection{Limit sets of Kleinian groups}

Hereafter, $\mathbf{H}^{n}$ implies $\mathbf{H}_{\mathbf{R}}^{n}$ or $\mathbf{H}_{\mathbf{C}}^{n}$, and $\mathrm{Isom}^{+} \mathbf{H}^{n}$ implies the group of all orientation-preserving isometries with respect to the hyperbolic metric.

We classify $A \in \mathrm{Isom}^{+} \mathbf{H}^{n}$ into three types. If $A$ fixes a point in $\mathbf{H}^{n}$, it is called elliptic. If $A$ has no fixed point in $\mathbf{H}^{n}$ and fixes only one point in $\partial \mathbf{H}^{n}$, it is called parabolic. If $A$ has no fixed point in $\mathbf{H}^{n}$ and fixes two points in $\partial \mathbf{H}^{n}$, it is called loxodromic. These cases exhaust all possibilities.

A subgroup of Isom $^{+} \mathbf{H}^{n}$ is called discrete if it is a discrete subset of Isom $^{+} \mathbf{H}^{n}$. A discrete subgroup of Isom $^{+} \mathbf{H}^{n}$ is called a Kleinian group. A Kleinian group $G$ acts on $\mathbf{H}^{n}$ properly discontinuously, that is, for any compact set $K$ in $\mathbf{H}^{n}$ there exist only finitely many elements $g$ of $G$ that satisfy $g(K) \cap K \neq \emptyset$. The quotient space $\mathbf{H}^{n} / G$ is considered as a hyperbolic $n$ dimensional orbifold.

For a Kleinian group $G \subset \operatorname{Isom}^{+} \mathbf{H}^{n}$, we consider the orbit $G(p)=\{g(p) \mid$ $g \in G\}$ of any point $p$ in $\mathbf{H}^{n}$ under the action of $G$. Since $G$ acts on $\mathbf{H}^{n}$ properly discontinuously, $G(p)$ has accumulation points only on $\partial \mathbf{H}^{n}$. They are called the limit points of $G$, and the set of all these points is called the limit set of $G$, which is denoted by $\Lambda(G) . \quad \Lambda(G)$ is a closed set and does not depend on the reference point $p \in \mathbf{H}^{n}$.

We can classify limit points according to the way how $G(p)$ accumulates to the limit point.

Definition 2.1. We call a point $z \in \Lambda(G)$ a conical limit point if for a geodesic ray $l$ ending at $z$ and for a $\delta>0$, there exists a sequence of distinct elements $\left\{g_{n}\right\} \subset G$ such that the orbit $\left\{g_{n}(p)\right\}$ approximates $z$ inside $\delta$ neighborhood of the ray $l$.

The above definition of a conical limit point actually depends neither on the reference point $p \in \mathbf{H}^{n}$ nor on the geodesic ray $l$.

Definition 2.2. We call a point $z \in \Lambda(G)$ a horospherical limit point if any horoball at $z$ contains a point of the orbit $G(p)$.

The above definition of a horospherical limit point also does not depend on the reference point $p \in \mathbf{H}^{n}$. A conical limit point is a horospherical limit point.

\subsection{Geometrically finite groups}

We explain geometrically finite Kleinian groups in $\mathbf{H}^{n}$. Let $G \subset \mathrm{Isom}^{+} \mathbf{H}^{n}$ be a Kleinian group and $G_{z}$ the stabilizer of a $z \in \partial \mathbf{H}^{n}$, i.e., $G_{z}=\{g \in G \mid$ $g(z)=z\}$.

Definition 2.3. A parabolic fixed point $z$ is called a cusped parabolic fixed point if $(\Lambda(G) \backslash\{z\}) / G_{z}$ is compact.

For a Kleinian group $G$, the convex hull of $\Lambda(G)$, denoted by $H_{G}$, is the minimal 
closed convex subset of $\mathbf{H}^{n}$ that contains the set of points on geodesics connecting any two points of $\Lambda(G)$.

Definition 2.4. We call the set $C_{G}=H_{G} / G$ the convex core of $G$. If $C_{G}$ is compact, $G$ is called convex co-compact.

Definition 2.5. We say that a Kleinian group $G$ is a geometrically finite group if the limit set $\Lambda(G)$ entirely consists of conical limit points and cusped parabolic fixed points. A Kleinian group which is not a geometrically finite group is called a geometrically infinite group.

For an $\varepsilon>0$, we divide $C_{G}$ into two parts:

1. $\left(C_{G}\right)_{(0, \varepsilon)}=\left\{p \in C_{G} \mid\right.$ there exists a non-trivial closed curve in $C_{G}$ passing through $p$ whose length is less than $\varepsilon\}$.

2. $\left(C_{G}\right)_{[\varepsilon, \infty)}=C_{G} \backslash\left(C_{G}\right)_{(0, \varepsilon)}$.

It is known that there exist equivalent conditions for geometric finiteness defined above (cf. Apanasov [1], Apanasov [2], Apanasov-Xie [3], Matsuzaki-Taniguchi $[8])$.

TheOREM 2.1. For a Kleinian group $G \subset \mathrm{Isom}^{+} \mathbf{H}^{n}$, the following conditions are equivalent:

1. $G$ is geometrically finite, i.e., the limit set $\Lambda(G)$ entirely consists of conical limit points and cusped parabolic fixed points;

2. for some (any) $\varepsilon>0$, $\varepsilon$-neighborhood of $C_{G}$ has a finite hyperbolic volume;

3. for some (any) $\varepsilon>0,\left(C_{G}\right)_{[\varepsilon, \infty)}$ is compact.

If a geometrically finite group $G$ does not have a parabolic transformation, then $C_{G}=\left(C_{G}\right)_{[\varepsilon, \infty)}$ for some $\varepsilon$. It is also known that there exist equivalent conditions for a geometrically finite group without parabolic transformations (cf. Matsuzaki-Taniguchi [8]).

THEOREM 2.2. For a Kleinian group $G \subset \operatorname{Isom}^{+} \mathbf{H}^{n}$, the following conditions are equivalent:

1. $G$ is a geometrically finite group without parabolic transformations, i.e., $\Lambda(G)$ entirely consist of conical limit points;

2. $G$ is convex co-compact;

3. $\Lambda(G)$ entirely consists of horospherical limit points.

\section{Group completions and limit sets}

In this section, we discuss a map from the group completion $\partial G$ to the limit set $\Lambda(G)$, due to Floyd [5]. For convenience of the reader, we will present the proofs of some results. (See Proposition 3.1 and Theorem 3.1 below.)

Let $\left(X, d_{X}\right)$ and $\left(Y, d_{Y}\right)$ be metric spaces, two groups $G, H$ act on $\left(X, d_{X}\right)$ 
and $\left(Y, d_{Y}\right)$, respectively. A map $f: X \rightarrow Y$ is called G-equivariant if there exists a homeomorphism $\psi: G \rightarrow H$ such that

$$
\psi(g) \circ f(x)=f \circ g(x)
$$

holds for all $x \in X$ and for all $g \in G$.

Let $G$ be a finitely generated Kleinian group. We consider the Cayley graph $K(G, \sigma)$ for a given finite set $\sigma$ of generators. This is a 1-complex whose vertices are elements of $G$, and such that two vertices $a, b \in G$ are joined by an edge if and only if $a=b \gamma^{ \pm 1}$ for some generator $\gamma \in \sigma$. For a $g \in G$, the word length of $g$ is the number of generators $\gamma \in \sigma$ representing $g$. In general, a word length is not determined uniquely because of the existence of relations. So, we define by $|g|$ the minimal word length of $g \in G . \quad|g|$ depends on a set of generators. Hereafter, we use the minimal word length $|g|$ with respect to the fixed $\sigma$.

Definition 3.1. We define a distance $d_{c}$ on the Cayley graph $K(G, \sigma)$ as follows. For $a, b \in G$,

1. $d_{c}(a, b)=0$, if $a=b$;

2. $d_{c}(a, b)=\min \left\{|a|^{-2},|b|^{-2}\right\}$, if $a=b \gamma^{ \pm 1}$ for some $\gamma \in \sigma$;

3. $d_{c}(a, b)=\inf _{C} \sum_{k=1}^{n} d_{c}\left(c_{k-1}, c_{k}\right)$, for general $a, b \in G$, where the infimum is taken over all paths $C$ connecting $a$ and $b$ with vertices $a=c_{0}, c_{1}, \ldots$, $c_{n-1}, c_{n}=b$.

Then, $\left(K(G, \sigma), d_{c}\right)$ is a metric space and $K(G, \sigma)$ is completed to $\overline{K(G, \sigma)}$ as a metric space.

Definition 3.2. We call $\partial G=\overline{K(G, \sigma)} \backslash K(G, \sigma)$ the group completion of $G$.

For $a, b \in \partial G$, the metric $d_{c}$ is extended on $\overline{K(G, \sigma)}$ by defining $d_{c}(a, b)=$ $\lim _{n \rightarrow \infty} d_{c}\left(a_{n}, b_{n}\right)$, where $\left\{a_{n}\right\}$ and $\left\{b_{n}\right\}$ are Cauchy sequences converging to $a$ and $b$, respectively. $\partial G$ is regarded as the set of equivalent classes of Cauchy sequences.

Definition 3.3. We call a Cauchy sequence $\left\{g_{n}\right\} \subset G$ a shortest path if for all $n \in \mathbf{N}$, there exists $\gamma \in \sigma$ such that $g_{n+1}=g_{n} \gamma^{ \pm 1}$ and $\left|g_{n}\right|=n$.

The following proposition shows the existence of a shortest path.

Proposition 3.1 (Floyd [5]). For any $g \in \partial G$, there exists a shortest path $\left\{g_{n}\right\} \subset G$ such that $g_{n} \rightarrow g$ as $n \rightarrow \infty$.

Proof. For any $g \in \partial G$, there is a Cauchy sequence $\left\{h_{n}\right\} \subset G$ such that $h_{n} \rightarrow g$ as $n \rightarrow \infty$. We set $h_{n}=\gamma_{n, 1} \gamma_{n, 2} \cdots \gamma_{n,\left|h_{n}\right|}$, where $\gamma_{i, j} \in \sigma$.

Since $\left\{\gamma_{n, 1}\right\}_{n \in \mathbf{N}}$ is an infinite set and $\sigma$ is a finite set, there exists a $\gamma_{1}^{\prime} \in \sigma$ such that $\gamma_{n, 1}=\gamma_{1}^{\prime}$ for infinitely many $n$. Let $\left\{h_{n}^{1}\right\}_{n \in \mathbf{N}}$ be the subsequence of $\left\{h_{n}\right\}$ with $\gamma_{n, 1}=\gamma_{1}^{\prime}$ and put

$$
h_{n}^{1}=\gamma_{1}^{\prime} \gamma_{n, 2} \cdots \gamma_{n,\left|h_{n}^{1}\right|}
$$


Inductively, we may find $\gamma_{m}^{\prime} \in \sigma$ and a subsequence $\left\{h_{n}^{m}\right\}$ of $\left\{h_{n}^{m-1}\right\} \quad(m=1,2, \ldots)$ so that

$$
h_{n}^{m}=\gamma_{1}^{\prime} \gamma_{2}^{\prime} \cdots \gamma_{m}^{\prime} \gamma_{n, m+1} \cdots \gamma_{n,\left|h_{n}^{m}\right|} \quad(n=1,2, \ldots) .
$$

Put $g_{n}=\gamma_{1}^{\prime} \gamma_{2}^{\prime} \cdots \gamma_{n}^{\prime}$. Then, we may show that $\left\{g_{n}\right\}_{n \in \mathbf{N}}$ is a shortest path. First, we show that $\left|g_{m}\right|=m$ for any $m \in \mathbf{N}$. If $\left|g_{m}\right|<m$ for some $m \in \mathbf{N}$, then we have

$$
\begin{aligned}
\left|h_{n}^{m}\right| & =\left|\gamma_{1}^{\prime} \gamma_{2}^{\prime} \cdots \gamma_{m}^{\prime} \gamma_{n, m+1} \cdots \gamma_{n,\left|h_{n}^{m}\right|}\right| \\
& \leq\left|\gamma_{1}^{\prime} \gamma_{2}^{\prime} \cdots \gamma_{m}^{\prime}\right|+\left|\gamma_{n, m+1} \cdots \gamma_{n,\left|h_{n}^{m}\right|}\right| \\
& \leq\left|g_{m}\right|+\left(\left|h_{n}^{m}\right|-m\right)<\left|h_{n}^{m}\right| .
\end{aligned}
$$

This is a contradiction. Hence, $\left|g_{m}\right|=m$ for any $m \in \mathbf{N}$.

Finally, we will show $d_{c}\left(g_{n}, h_{n}\right) \rightarrow 0$ as $n \rightarrow \infty$. For any $\varepsilon>0$, we take a $M \in \mathbf{N}$ with $\sum_{k=M+1}^{\infty} \frac{1}{k^{2}}<\varepsilon$. From the definitions of $g_{n}$ and $h_{n}^{m}$, we have

$$
\begin{aligned}
d_{c}\left(g_{n}, h_{n}^{M}\right) & \leq d_{c}\left(g_{n}, g_{M}\right)+d_{c}\left(g_{M}, h_{n}^{M}\right) \\
& \leq \sum_{k=M+1}^{n} \frac{1}{k^{2}}+\sum_{k=M+1}^{\left|h_{n}^{M}\right|} \frac{1}{k^{2}} \leq 2 \sum_{k=M+1}^{\infty} \frac{1}{k^{2}}<2 \varepsilon,
\end{aligned}
$$

for any $n>M$. Since $\left\{h_{n}\right\}_{n \in \mathbf{N}}$ is a Cauchy sequence, there exists $N \in \mathbf{N}$ such that $d_{c}\left(h_{n}, h_{m}\right)<\varepsilon$ for any $n, m \geq N$. Thus, we conclude that

$$
d_{c}\left(g_{n}, h_{n}\right) \leq d_{c}\left(g_{n}, h_{n}^{N_{1}}\right)+d_{c}\left(h_{n}^{N_{1}}, h_{n}\right)<3 \varepsilon
$$

for any $n>\max \{M, N\}$. It completes the proof of the proposition.

It is known that there exists the following continuous mapping from $\partial G$ onto $\Lambda(G)$ if the orbit $G(o)$ satisfies a certain geometric condition.

THEOREM 3.1 (Floyd [5]). If there is a constant $k>0$ so that $2 \log |g|-k \leq$ $d_{h}(o, g(o))$ for any $g \in G$, then there is a continuous, G-equivariant surjection $f: \partial G \rightarrow \Lambda(G)$.

Proof. We use the ball model $\mathbf{B}_{\mathbf{R}}^{n}$ in the case of $\mathbf{H}_{\mathbf{R}}^{n}$ or the projective model $\mathbf{B}_{\mathbf{C}}^{n}$ in the case of $\mathbf{H}_{\mathbf{C}}^{n}$.

Fix $o \in \mathbf{H}^{n}$. We define $F: K(G, \sigma) \rightarrow \mathbf{H}^{n}$ as follows. For every $a \in G$, $F(a)=a(o)$. If $a=b \gamma^{ \pm 1}$ for some $\gamma \in \sigma$, then $F$ maps the edge $[a, b]$ in $K(G, \sigma)$ joining the vertices $a, b \in G$ to the hyperbolic geodesic connecting $a(o)$ and $b(o)$.

Since $2 \log |g|-k \leq d_{h}(o, g(o))$ for any $g \in G$,

$$
1-|g(o)| \leq 2 e^{k}|g|^{-2}
$$

because $d_{h}(o, g(o))=\log (1+|g(o)|)(1-|g(o)|)^{-1}$. 
If $a=b \gamma^{ \pm 1}$ for some $\gamma \in \sigma$, then $d_{c}(a, b)=\min \left\{|a|^{-2},|b|^{-2}\right\}$. We may assume $d_{c}(a, b)=|a|^{-2}$. Since $F([a, b])$ is the geodesic connecting from $a(o)=$ $b\left(\gamma^{ \pm 1}(o)\right)$ to $b(o)$, it is the image of the geodesic from $o$ to $\gamma^{ \pm 1}(o)$ by $b \in G$. Hence, from the triangle inequality, for any $z \in F([a, b])$ we have

$$
\begin{aligned}
d_{h}(o, a(o)) & \leq d_{h}(o, z)+d_{h}(z, a(o))=d_{h}(o, z)+d_{h}\left(o, b^{-1}(o)\right) \\
& \leq d_{h}(o, z)+M,
\end{aligned}
$$

where $M=\max \left\{d_{h}(o, \gamma(o)) \mid \gamma \in \sigma\right\}$. Thus,

$$
e^{-M} \leq 2(1-|a(o)|)(1-|z|)^{-1} .
$$

By using (3.1) and (3.2), we may estimate the Euclidean distance $|F(a)-F(b)|$ of $F(a), F(b)$ in $\mathbf{H}^{\mathbf{n}}$;

$$
\begin{aligned}
|F(a)-F(b)| & =|a(o)-b(o)| \\
& \leq \int_{F([a, b])}|d z| \leq \int_{F([a, b])} \frac{2 e^{M}\left(1-|a(o)|^{2}\right)}{1-|z|^{2}}|d z| \\
& \leq e^{M}\left(1-|a(o)|^{2}\right) d_{h}(a(o), b(o)) \leq 2 e^{M}(1-|a(o)|) M \\
& \leq \frac{4 e^{k+M} M}{|a|^{2}}=4 e^{k+M} M d_{c}(a, b) .
\end{aligned}
$$

If $a \neq b \gamma^{ \pm 1}$ for any $\gamma \in \sigma$, then for any $\varepsilon>0$ there exists a path $C$ connecting $a, b$ such that $\sum_{i=1}^{n} d_{c}\left(c_{i-1}, c_{i}\right) \leq d_{c}(a, b)+\varepsilon$, where $a=c_{0}, c_{1}, \ldots, c_{n-1}, c_{n}=b$ are the vertices in $C$. Applying (3.3) to $c_{i-1}, c_{i}(i=1,2, \ldots, n)$, we have

$$
\begin{aligned}
|F(a)-F(b)| & \leq \sum_{i=1}^{n} \mid F\left(c_{i-1}-F\left(c_{i}\right) \mid\right. \\
& \leq 4 e^{k+M} \sum_{i=1}^{n} d_{c}\left(c_{i-1}, c_{i}\right) \\
& \leq 4 e^{k+M} M d_{c}(a, b)+4 e^{k+M} M \varepsilon .
\end{aligned}
$$

Hence, we conclude that

$$
|F(a)-F(b)| \leq 4 e^{k+M} M d_{c}(a, b)
$$

and $F: K(G, \sigma) \rightarrow \mathbf{H}^{n}$ is Lipschitz continuous. Thus, it has a continuous extension $F: \overline{K(G, \sigma)} \rightarrow \overline{\mathbf{H}^{n}}$. Put $f=\left.F\right|_{\partial G}$. Since $\Lambda(G)$ is the set of accumulation points and $\overline{K(G, \sigma)}$ is totally bounded, $f(\partial G)=\Lambda(G)$. Thus, $f$ is our desired mapping.

\section{Word length and limit sets}

We will study a relation of the word length and the limit sets. Let $G$ be a finitely generated Kleinian group. Fix a generating set $\sigma$ of $G$ and a point 
$o \in \mathbf{H}^{n}$. Let $|g|$ be the minimal word length of $g \in G$ with respect to $\sigma$. We define $\alpha(G)$ by

$$
\alpha(G)=\sup \left\{\alpha \mid \sup _{g \in G}\left\{\frac{|g|^{\alpha}}{e^{d_{h}(o, g(o))}}\right\}<+\infty\right\} .
$$

It is easily seen that $\alpha(G)$ does not depend on the set of generators and the reference point $o \in \mathbf{H}^{n}$. For a geometrically finite Kleinian group $G$, the following results on $\alpha(G)$ are known.

THEOREM 4.1 (Floyd [5]). Let $G$ be a finitely generated Kleinian group.

1. If $G$ is a geometrically finite group with parabolic transformations, then $\alpha(G)=2$.

2. If $G$ is a geometrically finite group without parabolic transformations, then $\alpha(G)=+\infty$.

Remark 4.1. In [5, Lemma in p. 213], Floyd showed that if $G$ is convex cocompact, then there exist constants $C_{1}, C_{2}>0$ such that

$$
C_{1}|g| \leq d_{h}(o, g(o)) \leq C_{2}|g|
$$

for all $g \in G$. Generally, if $G$ is finitely generated, then an inequality

$$
d_{h}(o, g(o)) \leq M|g|
$$

holds with $M=\max \left\{d_{h}(o, \gamma(o)) \mid \gamma \in \sigma\right\}$ by the triangle inequality. Therefore, we see that the inequality (4.1) holds for any $g \in G$ if $\alpha(G)=\infty$.

Remark 4.2. Suppose that a finitely generated Kleinian group $G$ has a parabolic transformation $g$. Then, from the fomula of the hyperbolic distance we see that there exist constants $k_{1}, k_{2}>0$ such that

$$
2 \log \left|g^{n}\right|-k_{1} \leq d_{h}\left(o, g^{n}(o)\right) \leq 2 \log \left|g^{n}\right|+k_{2}
$$

for all $n \in \mathbf{N}$. Thus, we verify that $\alpha(G) \leq 2$.

The author does not know whether the converse of the first statement of Theorem 4.1 is true or not. However, we may show that the converse of the second statement is valid.

THEOREM 4.2. Let $G$ be a finitely generated Kleinian group. Then, the following conditions are equivalent:

1. $G$ is convex co-compact;

2. $\alpha(G)>2$;

3. $\alpha(G)=+\infty$.

Hence, $\alpha(G)=2$ is a necessary and sufficient condition for $G$ to have a parabolic transformation when $G$ is a geometrically finite group. 
Proof. (1) $\Rightarrow$ (3) follows from Theorem 4.1, and (3) $\Rightarrow(2)$ is clear.

We will show $(2) \Rightarrow(1)$. By Theorem 2.2, it suffices to show that $\Lambda(G)$ entirely consists of horospherical limit points. Since $\alpha(G)>2$, there exist an $\varepsilon>0$ and a $k>0$ such that

$$
(2+\varepsilon) \log |g|-k \leq d_{h}(o, g(o))
$$

for all $g \in G$. Therefore, from Theorem 3.1 there is a continuous $G$-equivariant surjection $f: \partial G \rightarrow \Lambda(G)$. For each $z \in \Lambda(G)$, we may take $g \in \partial G$ so that $f(g)=z$. By Proposition 3.1, there exists a shortest path $\left\{g_{m}\right\} \subset G$ such that $g_{m} \rightarrow g$ with respect to $d_{c}$. Then we have $g_{m}(o) \rightarrow z$ with respect to the Euclidean distance by the construction of $f$.

Here we use the upper half-space model $\mathbf{H}_{\mathbf{R}}^{n} \cong \mathbf{R}_{+}^{n}=\left\{x=\left(x_{1}, x_{2}, \ldots, x_{n}\right) \in\right.$ $\left.\mathbf{R}^{n} \mid x_{n}>0\right\}$ with the metric $|d x| / x_{n}$. As for the case of the complex hyperbolic space, the following argument also works and prove Theorem 4.2.

Taking $h \in \mathrm{Isom}^{+} \mathbf{H}^{n}$ such that $h(o)=j=(0, \ldots, 0,1)$ and $h(z)=\infty$, we may assume that $\left|g_{m}(j)\right| \rightarrow \infty$ for the shortest path $\left\{g_{m}\right\} \subset G$. In order to show that there exists a point of the orbit $G(j)$ in any horoball $\left\{x \in \mathbf{R}^{n} \mid x_{n} \geq t\right\}$ $(t>0)$, we have only to prove the following lemma and we will show Theorem 4.2.

Lemma 4.1. Let $G$ be a finitely generated Kleinian group with $\alpha(G)>2$. Suppose that there exists a shortest path $\left\{g_{m}\right\} \subset G$ such that $\left|g_{m}(j)\right| \rightarrow \infty$ as $m \rightarrow \infty$ for $j=(0,0, \ldots, 0,1) \in \mathbf{H}^{n}$. Then, if $g_{m}(j)=\left(x_{m, 1}, x_{m, 2}, \ldots, x_{m, n}\right)$, $\left\{x_{m, n}\right\}_{m \in \mathbf{N}}$ is not bounded.

Proof. Let $\tilde{x}_{m}=\left(x_{m, 1}, x_{m, 2}, \ldots, x_{m, n-1}, 0\right)$. If there exists a $P>0$ such that $\left|\tilde{x}_{m}\right|<P$ for infinitely many $m$, then $\left\{x_{m, n}\right\}$ is unbounded since $\left|g_{m}(j)\right| \rightarrow \infty$ as $m \rightarrow \infty$.

Suppose that $\lim _{m \rightarrow \infty}\left|\tilde{x}_{m}\right|=\infty$ and $\left|x_{m, n}\right|<C(m \in \mathbf{N})$ for some constant $C>0$. From the definition of the shortest path, we see that an inequality $d_{h}\left(g_{m-1}(j), g_{m}(j)\right) \leq M$ holds for $M=\max \left\{d_{h}(j, \gamma(j)) \mid \gamma \in \sigma\right\}$. Since

$$
\begin{aligned}
& d_{h}\left(g_{m-1}(j), g_{m}(j)\right)= 2 \log \left(\sqrt{\left|\tilde{x}_{m-1}-\tilde{x}_{m}\right|^{2}+\left(x_{m-1, n}+x_{m, n}\right)^{2}}\right. \\
&\left.+\sqrt{\left|\tilde{x}_{m-1}-\tilde{x}_{m}\right|^{2}+\left(x_{m-1, n}-x_{m, n}\right)^{2}}\right) \\
&-\log \left(4 x_{m-1, n} x_{m, n}\right) \\
& \geq 2 \log \left|\tilde{x}_{m-1}-\tilde{x}_{m}\right|-\log x_{m-1, n} x_{m, n},
\end{aligned}
$$

we have

$$
\left|\tilde{x}_{m-1}-\tilde{x}_{m}\right| \leq e^{M / 2} \sqrt{x_{m-1, n} x_{m, n}} .
$$

If $g_{0}(j)=j=(0, \ldots, 0,1)$, then $\left|\tilde{x}_{0}\right|=0$ and 


$$
\begin{aligned}
\left|\tilde{x}_{m}\right|=\left|\tilde{x}_{m}-\tilde{x}_{o}\right| & \leq \sum_{i=1}^{m}\left|\tilde{x}_{i}-\tilde{x}_{i-1}\right| \leq e^{M / 2} \sum_{i=1}^{m} \sqrt{x_{i-1, n} x_{i, n}} \\
& \leq e^{M / 2} \sum_{i=1}^{m} \frac{x_{i-1, n}+x_{i, n}}{2} \leq e^{M / 2} \sum_{i=0}^{m} x_{i, n} .
\end{aligned}
$$

Since $\left|\tilde{x}_{m}\right| \rightarrow+\infty$, we have

$$
\sum_{i=0}^{m} x_{i, n} \rightarrow+\infty \quad(m \rightarrow \infty) .
$$

On the other hand, for some constant $A>0$ we have

$$
\begin{aligned}
d_{h}\left(j, g_{m}(j)\right)= & 2 \log \left(\sqrt{\left|\tilde{x}_{m}\right|^{2}+\left(x_{m, n}+1\right)^{2}}+\sqrt{\left|\tilde{x}_{m}\right|^{2}+\left(x_{m, n}-1\right)^{2}}\right) \\
& -\log \left(4 x_{m, n}\right) \leq \log \left(\left|\tilde{x}_{m}\right|^{2}+\left(x_{m, n}+1\right)^{2}\right)-\log x_{m, n} \\
\leq & \log \left(\left|\tilde{x}_{m}\right|^{2}+(C+1)^{2}\right)-\log x_{m, n} \\
\leq & \log \left(A\left|\tilde{x}_{m}\right|^{2}\right)-\log x_{m, n} .
\end{aligned}
$$

Since $\alpha(G)>2$, we obtain

$$
(2+\varepsilon) \log m-k \leq d_{h}\left(j, g_{m}(j)\right) \leq \log \left(A\left|\tilde{x}_{m}\right|^{2}\right)-\log x_{m, n}
$$

Put

$$
\beta=\inf \left\{b \geq 0 \mid \sup _{m \in \mathbf{N}}\left\{\left(\sum_{i=0}^{m} x_{i, n}\right) / m^{b}\right\}<+\infty\right\} .
$$

Since $x_{m, n}<C$ for all $m \in \mathbf{N}, \beta \leq 1$. By the definition of $\beta$, there exists a constant $L>0$ such that

$$
\sum_{i=0}^{m} x_{i, n} \leq L m^{\beta+\varepsilon / 4}
$$

By (4.7), (4.10) and (4.11), we have

$$
e^{-k} \leq \frac{A e^{M}\left(\sum_{i=0}^{m} x_{i, n}\right)^{2}}{m^{2+\varepsilon} x_{m, n}} \leq \frac{A e^{M} L^{2} m^{2 \beta-2-\varepsilon / 2}}{x_{m, n}} .
$$

Now, we have for infinitely many $m$ of $\mathbf{N}$

$$
\frac{m^{2 \beta-2-\varepsilon / 4}}{x_{m, n}} \leq 1 \text {. }
$$

Indeed, if not, there exist an $N \in \mathbf{N}$ such that $x_{i, n}<i^{2 \beta-2-\varepsilon / 4}$ for all $i \geq N$. Because $\sum_{i=N}^{m} x_{i, n}<\sum_{i=N}^{m} i^{2 \beta-2-\varepsilon / 4}$ and (4.8), $\sum_{i=N}^{m} i^{2 \beta-2-\varepsilon / 4} \rightarrow \infty \quad(m \rightarrow \infty)$. Hence, $2 \beta-2-\varepsilon / 4 \geq-1$. 
If $2 \beta-1-\varepsilon / 4>0$, there exists a constant $B_{1}>0$ such that

$$
\sum_{i=0}^{m} x_{i, n} \leq C N+\int_{N}^{m+1} i^{2 \beta-2-\varepsilon / 4} d i<C N+B_{1} m^{2 \beta-1-\varepsilon / 4} .
$$

By the definition of $\beta$, we see that $2 \beta-1-\varepsilon / 4 \geq \beta$, and $\beta \geq 1+\varepsilon / 4$. This contradicts $\beta \leq 1$. that

If $2 \beta-1-\varepsilon / 4=0$, i.e., $\beta=(4+\varepsilon) / 8$, there exists a constant $B_{2}>0$ such

$$
\sum_{i=0}^{m} x_{i, n} \leq C N+\int_{N}^{m+1} i^{-1} d i<C N+B_{2} \log m
$$

Since

$$
\frac{\sum_{i=0}^{m} x_{i, n}}{m^{1 / 4}}<\frac{C N+B_{2} \log m}{m^{1 / 4}}<+\infty
$$

for all $m$, we have $\beta \leq 1 / 4$ from the definition of $\beta$. However, this contradicts $\beta=(4+\varepsilon) / 8$. Therefore, we verify that (4.13) holds for infinitely many $m \in \mathbf{N}$.

From (4.12) and (4.13), for infinitely many $m$ of $\mathbf{N}$,

$$
e^{-k} \leq A e^{M} L^{2} m^{-\varepsilon / 4} \text {. }
$$

A contradiction occurs as $m \rightarrow \infty$. This completes the proof of Lemma 4.1.

Let $\phi: G \rightarrow H$ be an isomorphism of two Kleinian groups. If $d_{h}(o, g(o))$ and $d_{h}(o, \phi(g)(o))$ are close to each other for any $g \in G$, one may expect that the two Kleinian group have similar properties.

COROllary 4.1. Let $\phi: G \rightarrow H$ be an isomorphism of two Kleinian groups, and let $G$ be convex co-compact. Then, the following conditions are equivalent:

1. $H$ is convex co-compact;

2. There exist $\varepsilon>0$ and $k>0$ such that

$$
(2+\varepsilon) \log d_{h}(o, g(o))-k \leq d_{h}(o, \phi(g)(o))
$$

for any $g \in G$;

3. There exist constants $C_{1}, C_{2}>0$ such that

$$
C_{1} d_{h}(o, g(o)) \leq d_{h}(o, \phi(g)(o)) \leq C_{2} d_{h}(o, g(o))
$$

for any $g \in G$.

Proof. Note that $G$ is finitely generated since $G$ is geometrically finite (cf. Apanasov [1], Apanasov [2], Matsuzaki-Taniguchi [8]). Let $\sigma$ be a generating set of $G$. Then $\phi(\sigma)$ is a generating set of $H$ and the minimal word length $|\phi(g)|$ of $\phi(g)(g \in G)$ with respect to $\phi(\sigma)$ is equal to $|g|$, the minimal word length of $g$ with respect to $\sigma$.

$(3) \Rightarrow(2)$ is clear. Assume (2). By Remark 4.1, there exist constants $P_{1}, Q_{1}>0$ such that

$$
P_{1}|g| \leq d_{h}(o, g(o)) \leq Q_{1}|g|
$$


for all $g \in G$. Hence

$$
\begin{aligned}
(2+\varepsilon) \log P_{1}|\phi(g)|-k & =(2+\varepsilon) \log P_{1}|g|-k \\
& \leq(2+\varepsilon) \log d_{h}(o, g(o))-k \leq d_{h}(o, \phi(g)(o)) .
\end{aligned}
$$

This implies $\alpha(H)>2$, and we conclude that $H$ is convex co-compact from Theorem 4.2.

Assume (1). There exist constants $P_{2}, Q_{2}>0$ such that

$$
P_{2}|\phi(g)| \leq d_{h}(o, \phi(g)(o)) \leq Q_{2}|\phi(g)|
$$

for all $g \in G$ by Remark 4.1. Since $|g|=|\phi(g)|$, we have

$$
P_{2} / Q_{1} d_{h}(o, g(o)) \leq d_{h}(o, \phi(g)(o)) \leq Q_{2} / P_{1} d_{h}(o, g(o)) .
$$

This implies (3).

Corollary 4.2 immediately follows from Corollary 4.1.

COROLlary 4.2. Let $G$ be convex co-compact and $H$ a geometrically infinite group. Suppose that there exists an isomorphism $\phi: G \rightarrow H$. Then, for any $\varepsilon>0$, there exists a $g \in G$ such that $d_{h}(o, \phi(g)(o))<(2+\varepsilon) \log d_{h}(o, g(o))$.

\section{REFERENCES}

[1] B. N. Apanasov, Discrete groups in Space and Uniformization Problems, Math. and Appl. 40, Kluwer Academic Publishers, Dordrecht, 1991.

[2] B. N. Apanasov, Deformation and Stability in complex hyperbolic geometry, Preprint 1997111, MSRI at Berkeley, 1997.

[ 3 ] B. N. Apanasov and X. XIE, Geometrically finite complex hyperbolic manifolds, Intern. J. Math. 8 (1997), 703-757.

[4] D. B. A. EpsteIn, Complex Hyperbolic Geometry, Analytical and geometric aspects of hyperbolic space (Coventry/Durhem, 1984), 93-111.

[5] W. J. FloYD, Group Completions and limit sets of Kleinian groups, Invent. Math. 57 (1980), 205-218.

[6] W. M. Goldman, Complex hyperbolic geometry, The Clarendon Press, Oxford University Press, Oxford Science Publications, 1999.

[7] P. J. Nicholls, The limit set of a discrete group of hyperbolic motions, Holomorphic functions and moduli 2, Springer, New York, 1988, 141-164.

[8] K. Matsuzaki and M. Taniguchi, Hyperbolic Manifolds and Kleinian Groups, The Clarendon Press Oxford University Press, Oxford Science Publications, 1998.

[9] P. TuKIA, On isomorphism of geometrically finite Kleinian groups, Publ. Math. IHES 61 (1985), 171-214.

[10] P. Tukia, A remark on a paper by Floyd, Holomorphic functions and moduli 2, Springer, New York, 1988, 165-172.

5-5-22 FUCHINOBEHONTYO

SaGamihara-SHI, Kanagawa 229-0002

JAPAN

e-mail: logarithm@nyc.odn.ne.jp 